\title{
MicroRNA-17-5p activates hepatic stellate cells through targeting of Smad7
}

\author{
Fujun Yu' ${ }^{1}$ Yong Guo², Bicheng Chen ${ }^{3}$, Peihong Dong ${ }^{1}$ and Jianjian Zheng ${ }^{3}$
}

A considerable amount of research has focused on the roles of microRNAs (miRNA) in the pathophysiology of liver fibrosis in view of their regulatory effects on hepatic stellate cell (HSC) functions, including proliferation, differentiation, and apoptosis. Recently, miR-17-5p was shown to promote cell proliferation and migration in liver. Transforming growth factor- $\beta 1$ (TGF- $\beta 1$ ) has been characterized as the master fibrogenic cytokine that stimulates HSC activation and promotes progression of liver fibrosis. The issue of whether miR-17-5p plays a role in TGF- $\beta 1$-induced hepatic fibrogenesis remains to be established. In this study, we demonstrated a dose-/time-dependent increase in miR-17-5p expression in TGF- $\beta 1$-treated HSCs. Enhanced miR-17-5p expression was additionally observed in $\mathrm{CCl}_{4}$-induced rat liver fibrosis. Inhibition of miR-17-5p led to suppression of HSC proliferation induced by TGF- $\beta 1$ without affecting cellular apoptosis. Notably, miR-17-5p was significantly associated with TGF- $\beta 1$-induced expression of type I collagen and $a$-SMA in HSC. Furthermore, Smad7, a negative regulator of the TGF- $\beta /$ Smad pathway, was confirmed as a direct target of miR-17-5p. Serum miR-17-5p levels were significantly higher in patients with cirrhosis, compared to healthy controls. Our results collectively indicate that miR-17-5p promotes HSC proliferation and activation, at least in part, via reduction of Smad7, supporting its potential utility as a novel therapeutic target for liver fibrosis.

Laboratory Investigation (2015) 95, 781-789; doi:10.1038/labinvest.2015.58; published online 27 April 2015

Liver fibrosis represents a sustained wound healing response of liver to chronic injury characterized by accumulation of extracellular matrix (ECM) components, including collagen I. ${ }^{1,2}$ Accumulation of ECM distorts the normal architecture of liver by forming fibrous scars, eventually leading to cirrhosis. ${ }^{3}$ Hepatic stellate cells (HSC), the main producers of ECM in the fibrotic liver, play an essential role in liver fibrosis development. ${ }^{4}$ Following continued liver injury, quiescent HSCs transdifferentiate into myofibroblast-like cells, as illustrated by expression of smooth muscle $\alpha$-actin ( $\alpha$-SMA) and enhanced ECM production. ${ }^{5}$ In addition, upon response to fibrogenic stimuli, HSCs may lose their vitamin A stores and become proliferative and fibrogenic. Transforming growth factor- $\beta 1$ (TGF- $\beta 1$ ), one of the major profibrogenic mediators, activates transdifferentation of HSCs through the Smad2/3 pathway. ${ }^{6,7}$ However, the regulatory mechanisms of liver fibrosis are yet to be completely elucidated.

MicroRNAs (miRNAs) are endogenous, short (20-22 nucleotide) non-coding RNAs that post-transcriptionally regu- late gene expression by blocking translation at the initiation or post-initiation steps, inducing mRNA deadenylation and decay. ${ }^{8}$ miRNAs are involved in various biological processes, such as development and differentiation, immune response, metabolism, cell proliferation and apoptosis. ${ }^{9}$ Emerging studies have revealed a vital role of miRNAs in the pathogenesis and progression of liver fibrosis. For example, ectopic miR-21 stimulates extracellular signal-regulated kinase 1 (ERK1) signaling in HSCs and induces hepatocyte epithelial-mesenchymal transition (EMT) by targeting sprouty2 (SPRY2) or hepatocyte nuclear factor $4 \alpha(\mathrm{HNF} 4 \alpha){ }^{10}$ Overexpression of miR-29b suppresses cell viability and expression of $\alpha$-SMA in activated HSCs. ${ }^{11}$ Previously, we demonstrated that curcumin upregulates miR-29b expression, leading to silencing of DNA methyltransferase 3b (DNMT3b) and loss of phosphatase and tensin homolog deleted on chromosome 10 (PTEN) methylation, which contributes to suppression of activated HSCs. ${ }^{12}$ These findings clearly suggest that miRNAs act as HSC regulators and play diverse roles in hepatic fibrosis.

${ }^{1}$ Department of Infectious Diseases; The First Affiliated Hospital of Wenzhou Medical University, Wenzhou, Zhejiang, China; ${ }^{2}$ Institute of Organ Transplantation; The First Affiliated Hospital of Wenzhou Medical University, Wenzhou, Zhejiang, China and ${ }^{3}$ Key Laboratory of Surgery, The First Affiliated Hospital of Wenzhou Medical University, Wenzhou, Zhejiang, China

Correspondence: Dr P Dong, Department of Infectious Diseases, the First Affiliated Hospital of Wenzhou Medical University, No.2 fuxue lane, Wenzhou, Zhejiang, P.R. China or J Zheng, Key Laboratory of Surgery, the First Affiliated Hospital of Wenzhou Medical University, No.2 fuxue lane, Wenzhou, Zhejiang, P.R. China.

E-mail: dongpeihong111@163.com or120378196@qq.com

Received 11 December 2014; revised 26 February 2015; accepted 2 April 2015 
miR-17-5p, an important member of the miR-17-92 cluster, plays a crucial role in regulating cell functions, such as proliferation and migration, and is overexpressed in various cancers, including hepatocellular carcinoma (HCC). ${ }^{13,14}$ miR-17-5p is reported to synergistically trigger HCC development via its target PTEN. ${ }^{15}$ An earlier study by our group showed that high expression of serum miR-17-5p is associated with poor prognosis in HCC patients. ${ }^{16}$ However, the precise roles of miR-17-5p in liver fibrosis remain obscure at present. Here, we showed that miR-17-5p is increased in TGF- $\beta 1$-induced HSCs as well as in carbon tetrachloride $\left(\mathrm{CCl}_{4}\right)$-treated rat liver fibrotic tissues. Inhibition of miR-17-5p suppressed proliferation of HSCs, ECM production and $\alpha$-SMA expression induced by TGF- $\beta 1$. Furthermore, miR-17-5p activated HSCs through direct targeting of Smad7. Serum miR-17-5p levels were elevated in patients with cirrhosis. Identification of miR-17-5p as a novel miRNA involved in HSC activation and liver fibrosis supports its potential utility as a therapeutic target.

\section{MATERIALS AND METHODS}

\section{Cell Culture and Clinical Samples}

The rat HSC-T6 cell line was obtained from Research of the Chinese Academy of Medical Sciences, Beijing, China. Cells were maintained in DMEM containing 10\% fetal bovine serum, $100 \mathrm{U} / \mathrm{ml}$ penicillin $\mathrm{G}$ sodium salt and $100 \mathrm{U} / \mathrm{ml}$ streptomycin sulfate (Gibco, Carlsbad, CA, USA), and incubated at $37^{\circ} \mathrm{C}$ under an atmosphere of $5 \% \mathrm{CO}_{2}$. Exponentially growing cells were treated with $0,5,10$ and $15 \mathrm{ng} / \mathrm{ml}$ TGF- $\beta 1$ (R\&D Systems, Shanghai, China) or transfected with miR-17-5p mimics for $24 \mathrm{~h}$. Cells were harvested for RNA/miRNA isolation, and whole cell extracts subjected to western blot analysis.

Serum samples of 30 patients with cirrhosis and 30 healthy controls obtained between 2011 and 2012 were collected at the First Affiliated Hospital of Wenzhou Medical University, Wenzhou, China. Informed consent for use of blood samples was obtained from all participants, and the project approved by the Ethics Committee of the First Affiliated Hospital of Wenzhou Medical University.

\section{miRNA Transfection}

Cells were seeded in a 6-well plate at a density of $1 \times 10^{6}$ cells per well. The following day, the medium was replaced with Opti-MEM (Invitrogen, USA) and cells transfected with miR-17$5 \mathrm{p}$ inhibitor $(60 \mathrm{nM})$ and miRNA negative control (miR-NC) $(60 \mathrm{nM})$ (GenePharma, China) using Lipofectamine 2000 (Invitrogen, USA) for $24 \mathrm{~h}$. After $6 \mathrm{~h}$ of transfection, the medium was replaced with DMEM containing $10 \%$ FBS, and TGF- $\beta 1$ added at a concentration of $5 \mathrm{ng} / \mathrm{ml}$. Cells were additionally transfected with miR-17-5p mimics (60 nM) using Lipofectamine 2000 for $24 \mathrm{~h}$.

\section{$\mathrm{CCl}_{4}$ Liver Injury Model}

$\mathrm{CCl}_{4}$ was obtained from Sigma (St Louis, MO, USA) and liver fibrosis generated via 6-week treatment of adult male Sprague-
Dawley (180-220 g) rats with $\mathrm{CCl}_{4}\left(\mathrm{CCl}_{4} / \mathrm{olive}\right.$ oil, 1:1 [vol/vol] per kg body weight via intraperitoneal injection twice weekly) as described previously. ${ }^{17}$ Twenty rats were randomly divided into two groups. Rats in Group $1(n=10)$ received twiceweekly injections of olive oil (vehicle control) while those in Group $2(n=10)$ received twice-weekly injections of $\mathrm{CCl}_{4}$ plus oral PBS $\left(\mathrm{CCl}_{4}\right.$-treated rats). Animals were provided by the Experimental Animal Center of Wenzhou Medical University. The experimental protocol was approved by the Animal Care and Use Committee of the University. Rats were sacrificed after the last $\mathrm{CCl}_{4}$ injection and liver tissues harvested for further analysis. Tissues were used for hematoxylin and eosin $(\mathrm{H} \& \mathrm{E})$ and Masson staining following fixation with $10 \%$ formalin.

\section{Immunohistochemistry}

Liver tissues were fixed in $10 \%$ neutral buffered formalin solution, embedded in paraffin, and stained for routine histology. Sections were dewaxed in xylene and dehydrated in alcohol. Antigen retrieval was achieved by microwaving in citric saline for $15 \mathrm{~min}$. Endogenous peroxidase activity was blocked via hydrogen peroxide pretreatment for $15 \mathrm{~min}$ and further blocked using an avidin/biotin blocking kit (3 drops/ section for $20 \mathrm{~min}$, with a phosphate-buffered saline (PBS) wash between stages). Sections were incubated with primary antibody against $\alpha$-SMA $(1: 100)$ for $20 \mathrm{~h}$ at $4{ }^{\circ} \mathrm{C}$, followed by incubation with biotinylated secondary antibody for $60 \mathrm{~min}$ at room temperature. $\alpha$-SMA expression was visualized with 3,3'-diaminobenzidine tetrahydrochloride (DAB) staining. Slides were counterstained with hematoxylin before dehydration and mounting, and $\alpha$-SMA-positive areas within the fibrotic region observed. Quantitative analysis was performed using five fields for each liver slice.

\section{Quantitative Real-Time PCR}

Total RNA was extracted from serum and cells with Trizol (Invitrogen, USA) and the miRNeasy Mini kit (Qiagen, Valencia, CA, USA). cDNA was synthesized using the ReverTra Ace qPCR RT Kit (Toyobo, Osaka, Japan) in accordance wtih the manufacturer's instructions. Gene expression was measured with real-time PCR using CDNA and SYBR Green real-time PCR Master Mix (Toyobo, Osaka, Japan). Primers for alpha-1 (I) collagen (Col1A1), $\alpha$-SMA, GAPDH and U6 were designed as described previously. ${ }^{18}$ To detect miR-17-5p and miR-238 expression, the RT reaction was performed using the TaqMan MicroRNA Assay (Applied Biosystems, Foster City, CA) according to the manufacturer's instructions. GAPDH and U6 snRNA (Applied Biosystems, Foster City, CA) levels were measured and used to normalize the relative abundance of mRNA and miRNA, respectively. Serum miR-17-5p expression was normalized against that of miR-238. ${ }^{19}$ The relative expression level $\left(2^{-\Delta \Delta \mathrm{Ct}}\right)$ of miRNA was calculated in accordance with a previous report. ${ }^{20}$ 


\section{Protein Extraction and Western Blot}

Protein concentrations in samples were determined with a BCA protein assay kit (Beyotime Biotechnology, Jiangsu, China). Total proteins $(30-50 \mu \mathrm{g})$ were separated with SDSPAGE and blotted onto a PVDF membrane (Millipore Corp, Billerica, MA, USA). After blocking, nitrocellulose blots were incubated for $1 \mathrm{~h}$ with primary antibodies diluted in TBS/ Tween-20 (0.075\%) containing 3\% Marvel. Rabbit polyclonal antibody against type I collagen (Abcam, Cambridge, MA, USA) diluted 1:2000 and mouse monocloonal antibodies against $\alpha$-SMA, Smad7 and $\beta$-actin (Santa Cruz, USA) diluted 1:1000 were used. Membranes were washed three times with TBS/Tween-20 (0.075\%) containing 3\% Marvel, followed by incubation with HRP-conjugated secondary antibodies (1:5000 dilution) at $37^{\circ} \mathrm{C}$ for $1 \mathrm{~h}$. The antigen-antibody complex was developed with enhanced chemiluminescence, exposed in the dark room and analyzed for integral absorbance (IA) of protein bands using quantitative software Quantity One 4.4.

\section{Cell Proliferation Assay}

Cells were seeded in a 96-well plate at a density of $1 \times 10^{3}$ cells per well, and transfected with miR-17-5p mimics, miR-17-5p inhibitor and miR-NC as described above. Cell proliferation was determined with the 3-(4,5-dimethylthiazol-2-yl)-2,5diphenyl-tetrazolium Bromide (MTT) assay in keeping with instructions of the MTT cell proliferation assay kit (Beyotime Institute of Biotechnology). Optical density (OD) was measured at $490 \mathrm{~nm}$ on a microplate reader (Bio-Rad 550, USA). All experiments were performed in triplicate and repeated at least three times.

\section{Apoptosis Analysis}

Quantification of apoptotic cells was performed with the Annexin-V-FITC Apoptosis Detection Kit (Bestbio, China), according to the manufacturer's instructions. Cells in the early stages of apoptosis were defined as Annexin-V-positive, PI-negative. Analyses were performed on a BD LSR flow cytometer (BD Biosciences) and experiments repeated three times.

\section{Cell Cycle Analysis}

To evaluate the mechanism underlying cell growth deficiency in miR-17-5p-transfected HSCs, the Cell Cycle and Apoptosis Analysis Kit was used (Beyotime, China) according to the manufacturer's instructions. Cells were harvested $24 \mathrm{~h}$ later, washed three times with cold PBS, and fixed in $70 \%$ ethanol in PBS at $-20^{\circ} \mathrm{C}$ for $24 \mathrm{~h}$. After fixation, cells were labeled with $0.5 \mathrm{ml}$ propidium iodide (PI) staining buffer containing $200 \mathrm{mg} / \mathrm{ml} \mathrm{RNase} \mathrm{A}$ and $50 \mu \mathrm{g} / \mathrm{ml}$ PI at $37^{\circ} \mathrm{C}$ for $40 \mathrm{~min}$ in the dark. Analyses were performed on a BD LSR flow cytometer (BD Biosciences) and experiments repeated at least three times.

\section{Luciferase Activity Assay}

Based on miRDB analysis, oligonucleotides containing the rat Smad7 3'UTR target sequence were annealed and cloned into the pMIR-Report ${ }^{\mathrm{TM}}$ Luciferase plasmid (Applied Biosystems) following the manufacturer's protocol to generate pMIRSmad7-17-5p. The Smad7 3'UTR target sequence for miR-17-5p (positions 1443-1449) was as follows: forward, $5^{\prime}$-AGCCCC GTCATAACAAAGGT- ${ }^{\prime}$ ' and reverse, 5' ${ }^{\prime}$-TCCTTTAGAAAA ACATTCAGCTAGG-3'. Empty vector pMIR was used as a negative control and pMIR-Report $\beta$-gal control plasmid employed for normalization of transfection. Cells were cultured in 24-well plates and transfected with $800 \mathrm{ng}$ pMIR-17-5p or pMIR together with $100 \mathrm{ng}$ pMIR- $\beta$-gal and $20 \mathrm{pmol}$ of miR-17-5p precursor or miR-NC (GenePharma, Shanghai, China), using Lipofectamine2000. Forty-eight hours after transfection, luciferase and $\beta$-gal activities were measured using the Dual-Light System (Applied Biosystems).

\section{Statistical Analysis}

Data from at least three independent experiments were expressed as means \pm s.d. Statistical analysis was performed using Student's $t$-test, with $P<0.05$ considered significant. All statistical analyses were performed with SPSS software (version 13; SPSS, Chicago, IL).

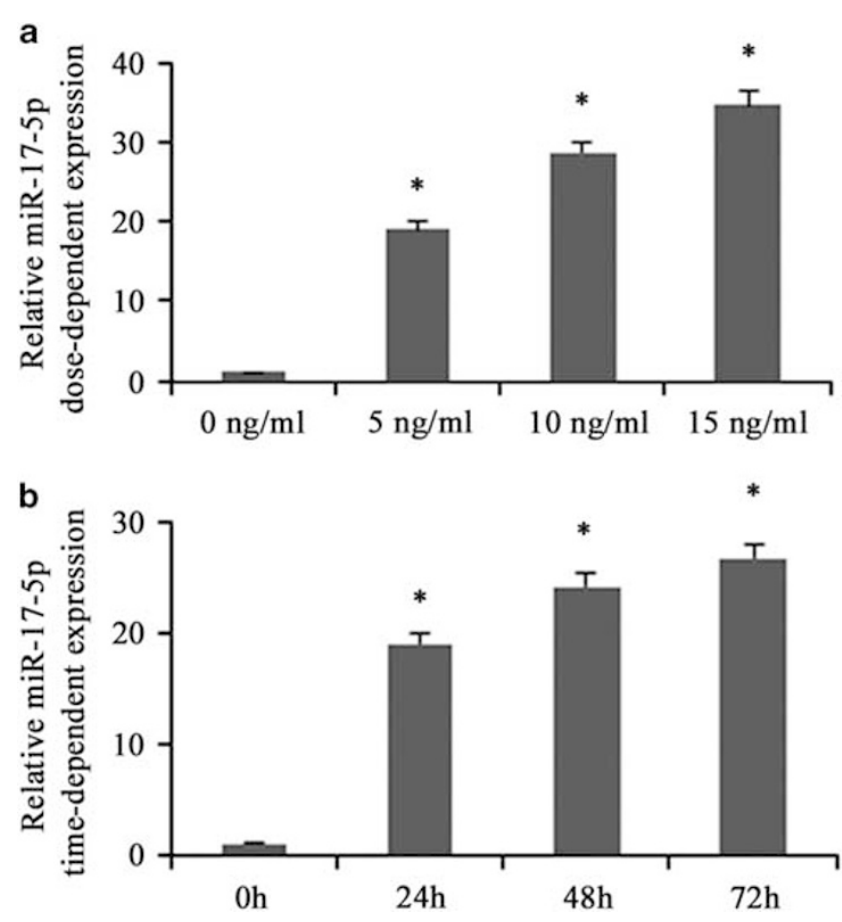

Figure 1 Upregulation of miR-17-5p expression in TGF- $\beta 1$-activated HSCs. (a) HSC-T6 cells were treated with TGF- $\beta 1(0,5,10$ and $15 \mathrm{ng} / \mathrm{ml})$ for $24 \mathrm{~h}$. miR-17-5p expression was upregulated in a dose-dependent manner by TGF- $\beta 1$. (b) HSC-T6 cells were treated with TGF- $\beta 1(5 \mathrm{ng} / \mathrm{ml})$ for $0,24,48$ and $72 \mathrm{~h}$, and miR-17-5p expression analyzed via real-time PCR. miR-17-5p expression was upregulated in a time-dependent manner by TGF- $\beta 1$. Each value represents the mean \pm s.d. of three experiments. ${ }^{*} P<0.05$, compared with the control. 
a

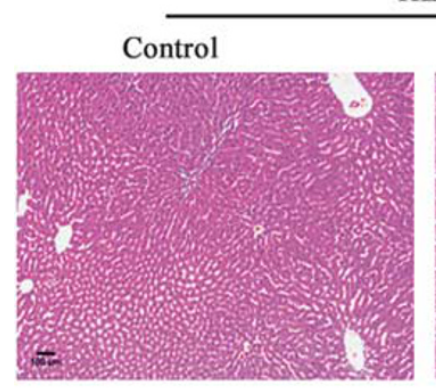

HE

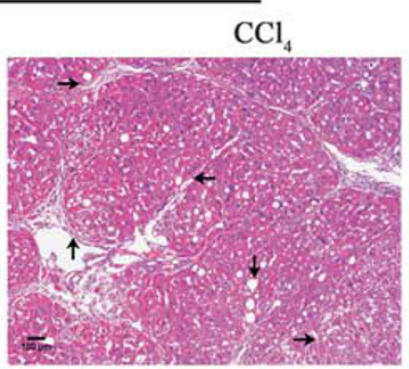

b

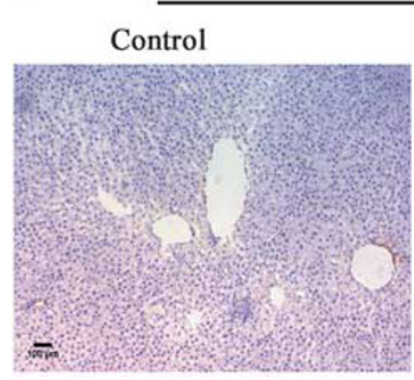

$\alpha$-SMA

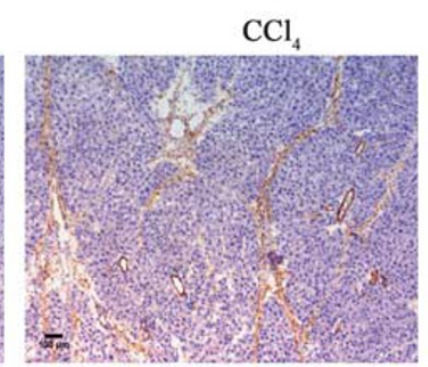

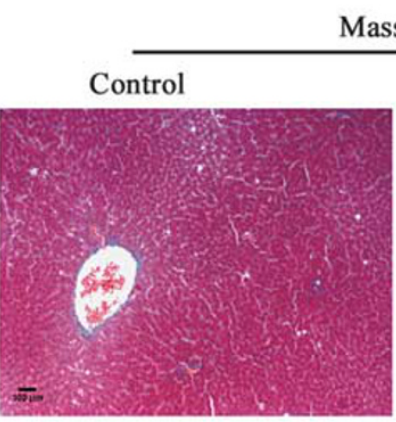

C

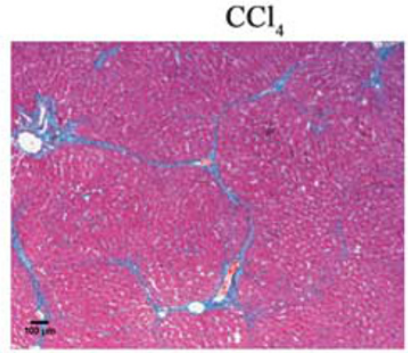

d

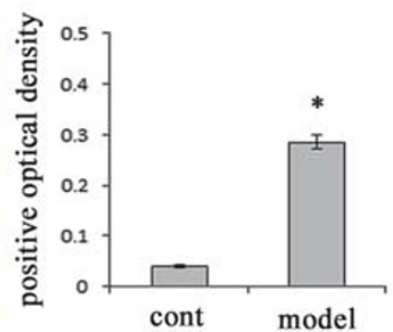

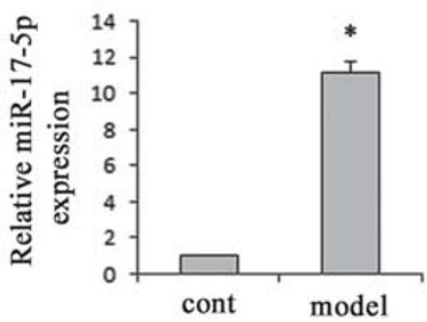

Figure 2 miR-17-5p expression in $\mathrm{CCl}_{4}$-treated hepatic fibrotic and control tissues. (a) Hepatic fibrotic and control tissues were detected by hematoxylin and eosin (H\&E) staining $(\times 100)$ and Masson staining $(\times 100)$, respectively. Black arrows indicate hepatic steatosis, necrosis and formation of regenerative nodules. (b) The $a$-SMA level was analyzed using immunohistochemistry in $\mathrm{CCl}_{4}$-treated hepatic fibrotic and control tissues $(\times 100)$. (c) Immunoexpression of a-SMA in hepatic fibrotic and control tissues. (d) Increased miR-17-5p expression in hepatic fibrotic tissues, compared with control tissues. Each value represents the mean \pm s.d. of three experiments. ${ }^{*} P<0.05$, compared with the control.

\section{RESULTS}

\section{miR-17-5p is Increased in TGF- $\beta 1$-Treated HSC and Liver Fibrotic Tissues}

Activation of HSCs is an important event in progression of liver fibrosis. TGF- $\beta 1$ has been characterized as the master fibrogenic cytokine stimulating HSC activation and collagen deposition. ${ }^{21}$ In our experiments, the rat HSC line, HSC-T6, was treated with different concentrations of TGF- $\beta 1$, and the effects on miR-17-5p expression examined. TGF- $\beta 1$ induced an increase in miR-17-5p expression in a dose-dependent manner, ranging from concentrations of 5 to $15 \mathrm{ng} / \mathrm{ml}$ (Figure 1a). miR-17-5p levels were additionally analyzed at $0,24,48$ and $72 \mathrm{~h}$ in TGF- $\beta 1$-treated HSCs. Expression was significantly increased with time, with the highest level observed at $72 \mathrm{~h}$ (Figure 1b), clearly indicating elevated levels of miR-17-5p in TGF- $\beta 1$-activated HSCs. To further establish expression of miR-17-5p in liver tissues from $\mathrm{CCl}_{4}$-treated rats, the severity of liver fibrosis was determined with $\mathrm{H} \& \mathrm{E}$ and Masson staining. $\mathrm{CCl}_{4}$ treatment caused prominent hepatic steatosis, necrosis, and formation of regenerative nodules in rat liver tissues (Figure 2a). Immunohistochemical images disclosed enhanced $\alpha$-SMA expression in $\mathrm{CCl}_{4}$-treated rats (Figure $2 \mathrm{~b}$ and $\mathrm{c}$ ). Moreover, compared with control liver, real-time PCR analysis demonstrated increased miR-17$5 \mathrm{p}$ expression in $\mathrm{CCl}_{4}$-treated liver (Figure 2d). Our data collectively suggest that miR-17-5p plays a significant role in liver fibrosis.

\section{miR-17-5p Promotes HSC Proliferation}

To ascertain whether miR-17-5p functions in TGF- $\beta 1$ mediated activation of HSCs, we examined its effects on HSC proliferation with the MTT assay. The results showed that miR-17-5p mimics significantly promote HSC proliferation and conversely, miR-17-5p inhibitors suppress cell proliferation induced by TGF- $\beta 1$ (Figure 3a). Generally, abnormal cell proliferation is related to apoptosis or alterations in the cell cycle. Accordingly, flow cytometric analyses were applied to examine effects of the miR-17-5p inhibitor on apoptosis and the cell cycle in HSC-T6. We observed no significant changes in the apoptosis rate between cells transfected with miR-17-5p inhibitor and those transfected with miR-NC (Figure 3b). However, cell cycle analysis revealed that the miR-17-5p inhibitor suppresses a proportion of cells in the $S$ phase and increases the number of cells in the G0/G1 phase (Figure 3c). These data suggest that miR-17-5p promotes cell proliferation via controlling transition from the G0/G1 to S phase.

\section{miR-17-5p Modulates TGF- $\beta 1-M e d i a t e d$ Collagen Expression}

To further establish the potential role of miR-17-5p in HSC activation, we examined its effects on collagen expression. Enhanced Col1A1 mRNA induced by TGF- $\beta 1$ was significantly decreased in the presence of the miR-17-5p inhibitor (Figure 4a). Similarly, the type I collagen protein level was 
a

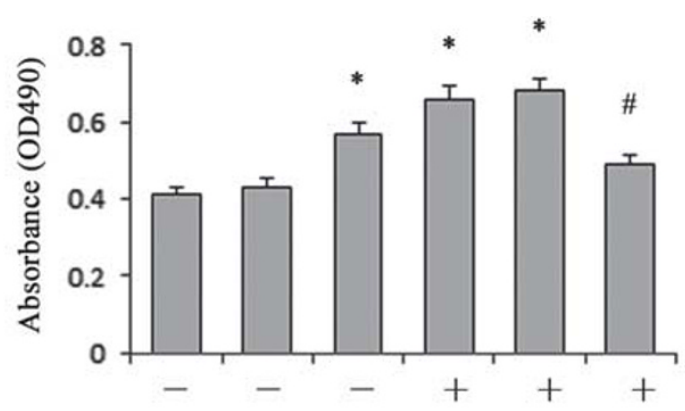

TGF- $\beta 1$

$\begin{array}{llll}- & + & -\end{array}$

miR-NC

miR-17-5p mimics

$\begin{array}{llllll}- & - & + & - & -\end{array}$

miR-17-5p inhibitor

b
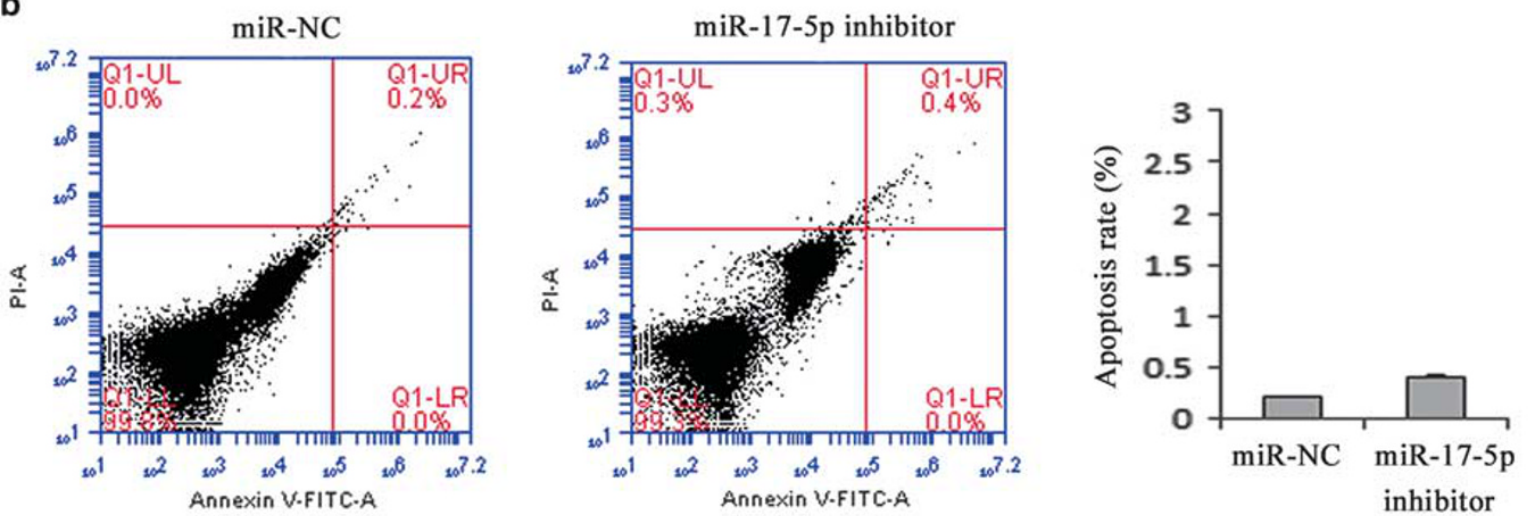

C

miR-NC
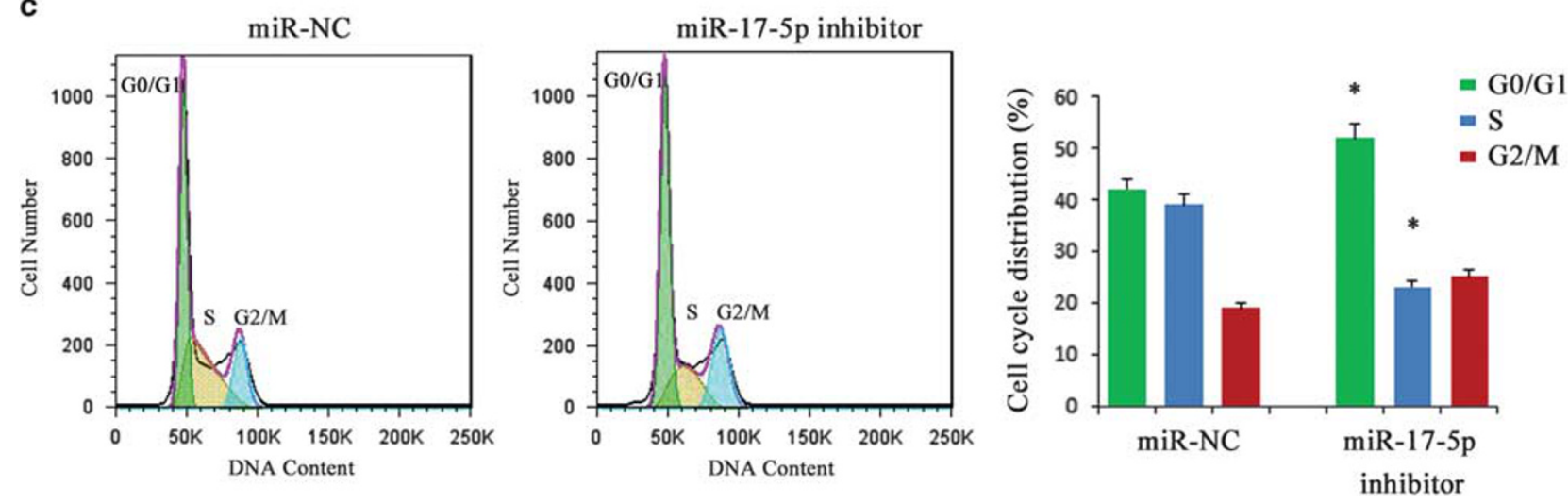

Figure 3 Effects of miR-17-5p inhibitor on cell proliferation, cell cycle and apoptosis in TGF- $\beta 1$-activated HSCs. (a) miR-17-5p mimics increased proliferation while miR-17-5p inhibitor inhibited proliferation of TGF- $\beta 1$-induced HSCs. Data were analyzed using the MTT assay. (b) Effect of miR-17-5p inhibitor on apoptosis in TGF- $\beta 1$-induced HSCs. (c) Effect of miR-17-5p inhibitor on the cell cycle in TGF- $\beta 1$-induced HSCs. Each value represents the mean \pm s.d. of three experiments. ${ }^{*} P<0.05$, compared with the control or miR-NC. ${ }^{*} P<0.05$, compared with the TGF- $\beta 1$ group.

markedly reduced by the miR-17-5p inhibitor in TGF- $\beta 1$ treated cells (Figure $4 \mathrm{~b}$ ), suggesting that miR-17-5p induces collagen expression in activated HSCs.

\section{HSC Transdifferentiation is Regulated by miR-17-5p}

HSC activation is characterized by enhanced cell proliferation, overproduction of ECM and de novo synthesis of $\alpha$-SMA. ${ }^{22}$ Inhibition of miR-17-5p has been shown to induce reduction of proliferation and type I collagen in TGF- $\beta 1$-treated cells. To examine the effect of miR-17-5p on $\alpha$-SMA expression, real-time PCR and immunoblot analysis were performed for detecting $\alpha$-SMA mRNA and protein in activated HSCs, respectively. The results of real-time PCR showed that the miR-17-5p inhibitor significantly inhibits the $\alpha$-SMA mRNA level in TGF- $\beta 1$-treated cells (Figure 4c). Consistent with mRNA data, immunoblot assays revealed that protein 


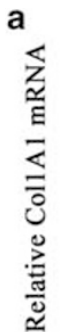

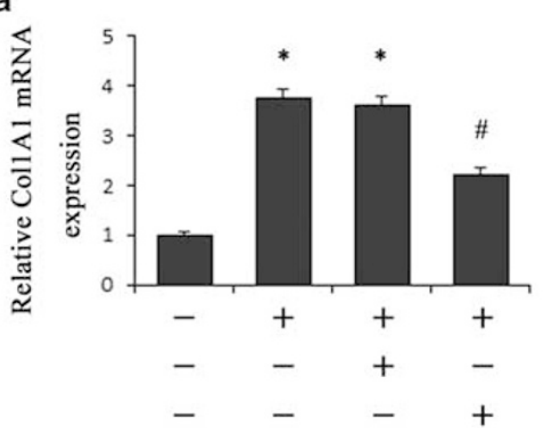

b

Type I collagen
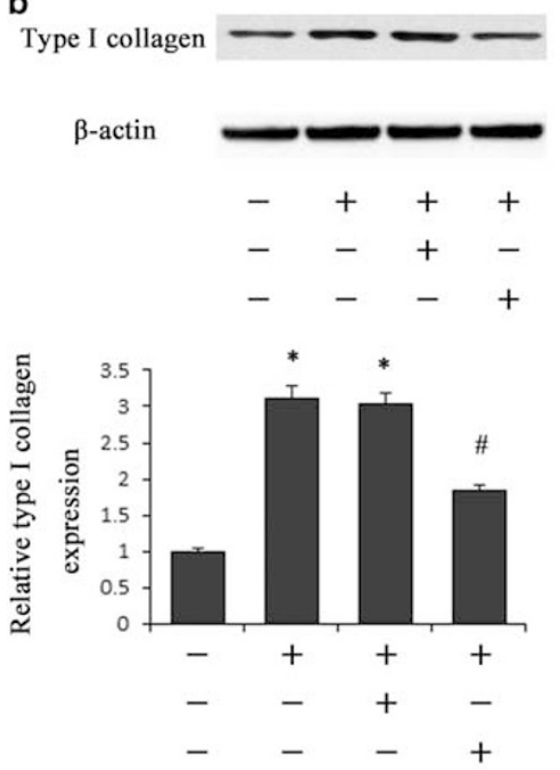

TGF- $\beta 1$

miR-NC

miR-17-5p inhibitor

TGF- $\beta 1$

$\operatorname{miR}-\mathrm{NC}$

miR-17-5p inhibitor

TGF- $\beta 1$

miR-NC

miR-17-5p inhibitor

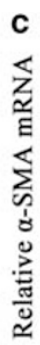

c

d
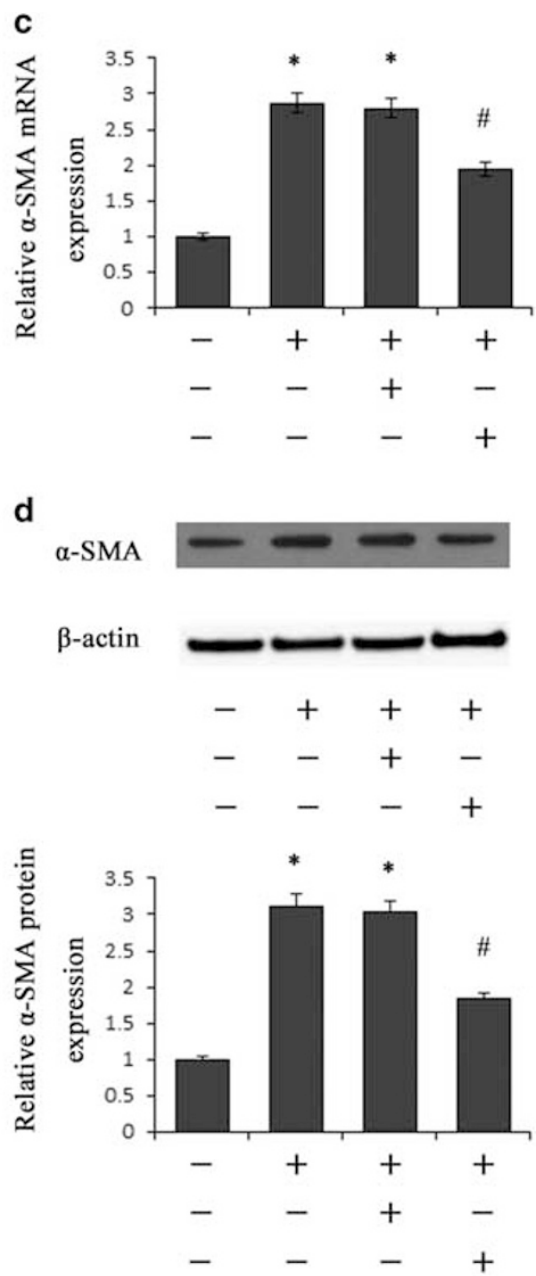

Figure 4 Type I collagen synthesis and HSC transdifferentiation are regulated by miR-17-5p. (a) Col1A1 mRNA expression in cells transfected with miR-17-5p inhibitor was analyzed with real-time PCR. (b) Type I collagen protein expression in cells transfected with miR-17-5p inhibitor was analyzed via Western blot using $\beta$-actin as the internal control. (c) $a$-SMA mRNA expression in cells transfected with miR-17-5p inhibitor was analyzed using realtime PCR. (d) $a$-SMA protein expression in cells transfected with miR-17-5p inhibitor was analyzed via Western blot, using $\beta$-actin as the internal control. Each value represents the mean \pm s.d. of three experiments. ${ }^{*} P<0.05$, compared with the control. ${ }^{\#} P<0.05$, compared with the TGF- $\beta 1$ group.

expression of $\alpha$-SMA is suppressed by the miR-17-5p inhibitor (Figure 4d).

\section{Smad7 is a Target of miR-17-5p}

To determine the mechanism by which miR-17-5p promotes HSC activation, bioinformatic analysis (miRDB) was employed to identify the potential targets of miR-17-5p. Among the miR-17-5p target genes, we focused on Smad7 due to its negative role in TGF- $\beta$ /Smad signaling. The $3^{\prime} \mathrm{UTR}$ of the Smad7 mRNA target region was cloned into pMIRREPORT $^{\mathrm{TM}}$ luciferase plasmid (Figure $5 \mathrm{a}$ and b). The construct was co-transfected into HSC-T6 cells, along with the miR-17-5p precursor or miR-NC. Co-transfection of $\beta$-gal reporter control plasmid was performed to monitor transfection efficiency. Notably, the miR-17-5p precursor induced significant reduction of luciferase activity driven by wild-type 3'UTR of Smad7, compared to miR-NC in HSCs but did not affect luciferase activities of mutant type Smad7 3' UTR and empty vector (Figure $5 \mathrm{c}$ ). To confirm these results, miR-17-5p mimics were transfected into HSC-T6 cells, and Smad7 mRNA and protein expression examined. Real-time PCR and western blot results showed significant downregulation of Smad7 mRNA and protein levels in cells transfected with miR-17-5p, compared with the control group (Figure $5 \mathrm{~d}$ and e). Furthermore, suppression of Smad7 by TGF- $\beta 1$ was blocked by the miR-17-5p inhibitor (Figure 6a and b). These findings collectively suggest that Smad7 is a direct target of miR-17-5p.

\section{miR-17-5p is Increased in Serum of Patients with Liver Cirrhosis}

Several recent reports have shown that miRNAs are detectable in serum and plasma in a sufficiently stable form to serve as biomarkers. $^{23,24}$ In view of the potential role of miR-17-5p in 


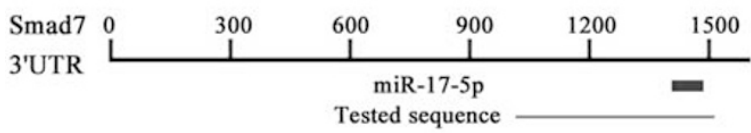

b

pMiR-17-5p (Positon 1443-1449 of Smad7 3'UTR)

$\begin{array}{lrl}\text { Smad7 3'UTR } & 5 ' \ldots \text { TAAAGAAAAGATGCACTTTGC. .. } \\ \text { rno-miR-17-5p } & 5^{\prime} \ldots \text {. . GACGUGACAUUCGUGAAAC. . }\end{array}$

Smad7 3'UTR Mut 5' ... TAAAgAaAAGATGCAGCCCGC. . . rno-miR-17-5p 5' ...GACGUGACAUUCGUGAAAC...

C

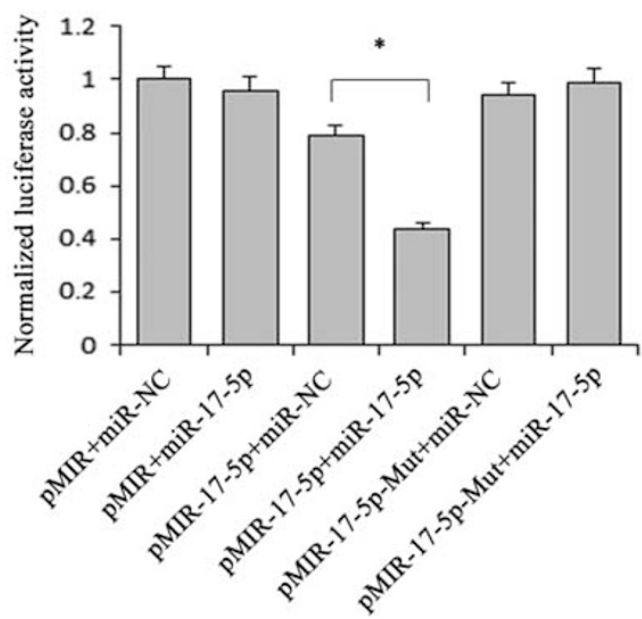

d
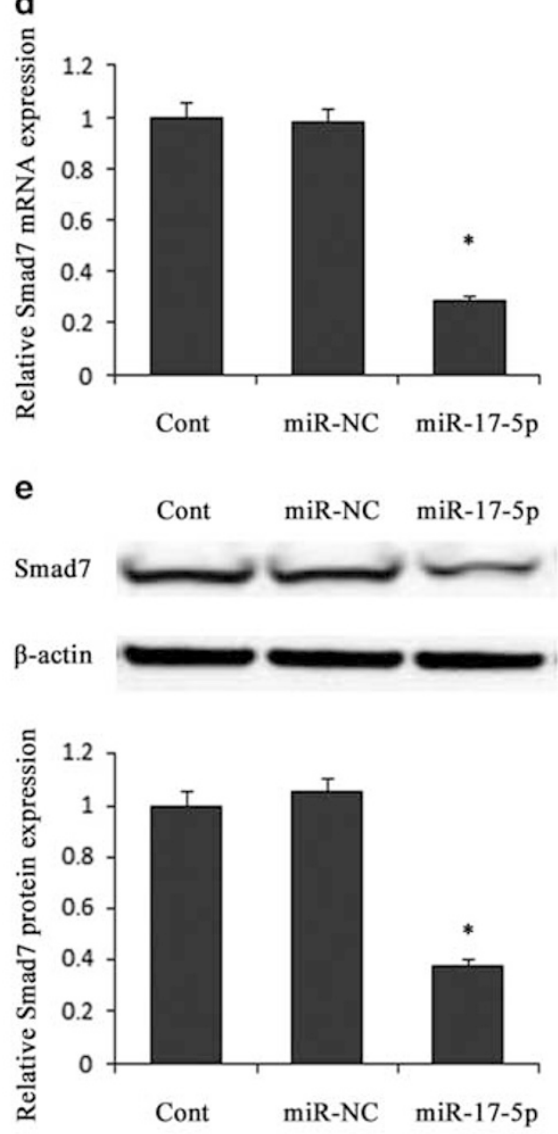

Figure 5 Interaction of miR-17-5p with the 3'UTR of Smad7 in HSCs. (a) Schematic representation of miRNA binding sites in the 3'UTR of Smad7 mRNA based on miRDB. The black box indicates miR-17-5p and 'tested sequence' signifies the region inserted into the luciferase reporter vector. (b) Predicted consequential pairing of the target region and miR-17-5p. According to the pairing sites, corresponding luciferase reporter vectors were designated pMIR-17-5p and pMIR-17-5p-Mut. (c) HSCs were transfected with pMIR (empty vector), pMIR containing wild-type miR-17-5p targeting sequence (pMIR-17-5p) and pMIR with mutated miR-17-5p target sequence (pMIR-17-5p-Mut). The graph depicts luciferase activity in cells transfected with pMIR-17-5p or pMIR-17-5p-Mut as well as those co-transfection with miR-17-5p precursor or miR-NC. ${ }^{*} P<0.05$. (d) Smad7 mRNA expression was analyzed in HSCs treated with miR-17-5p mimics or miR-NC using real-time PCR. (e) Protein expression of Smad7 in HSCs treated with miR-17-5p mimics or miR-NC was analyzed via western blot with $\beta$-actin as the internal control. Each value represents the mean \pm s.d. of three experiments. ${ }^{*} P<0.05$, compared with the control.

proliferation and activation of HSCs, miR-17-5p expression was examined in serum of liver cirrhosis patients. Notably, serum miR-17-5p levels were increased in patients with cirrhosis, compared to healthy controls (Figure 6c).

\section{DISCUSSION}

Under various conditions inducing liver injury, including hepatitis, alcohol abuse and exposure to toxic agents, liver fibrosis develops in many chronic liver diseases, resulting in cirrhosis, liver failure, and portal hypertension. No effective drugs that inhibit liver fibrosis are currently available. Activation of HSCs into a myofibroblast-like phenotype is considered the dominant event in liver fibrosis that contributes to ECM accumulation. ${ }^{25}$ TGF- $\beta 1$, the main profibrogenic mediator, accelerates the progression of liver fibrosis by triggering proliferation and differentiation of fibroblasts into myofibroblasts, a process known as HSC activation. ${ }^{26}$ Therefore, clarification of the TGF- $\beta 1$ and HSC activation pathways should reveal novel therapeutic targets to inhibit progression of liver fibrosis.

The present study aimed to determine whether miR-17-5p plays a role in regulating HSC activation. In vitro, miR-17-5p expression was upregulated in a dose/time-dependent manner in response to TGF- $\beta 1$. In vivo, miR-17-5p expression was elevated in liver fibrotic tissues, compared with control liver tissues, suggesting that the miR-17-5p level during liver fibrosis may be related to HSC activation. Data from the cell proliferation assay showed that suppression of miR-17-5p leads to significant inhibition of cell proliferation in TGF- $\beta 1$ treated HSCs, with an increase in the G0/G1 phase and reduction in the $S$ phase. However, miR-17-5p overexpression has no significant effect on cell apoptosis. Activated HSCs are 
a

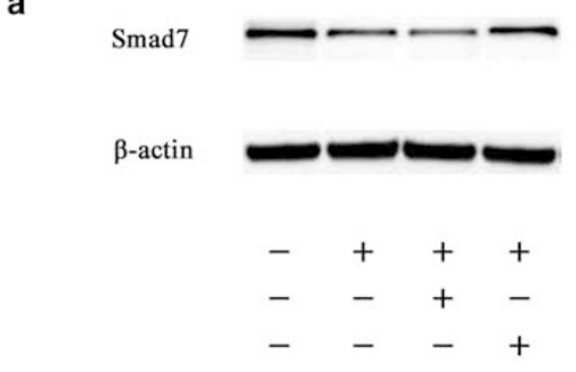

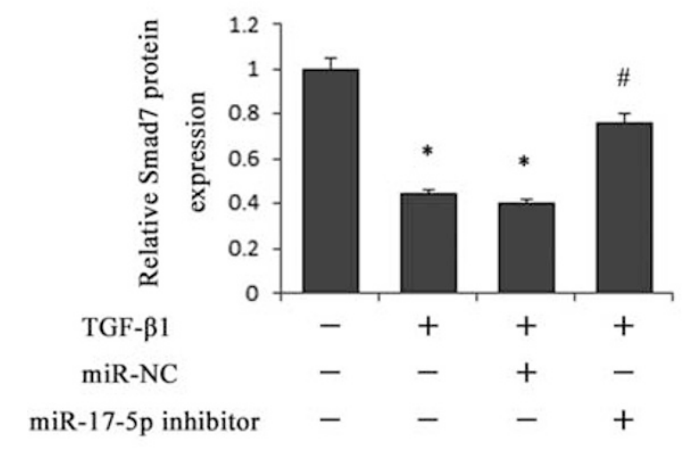

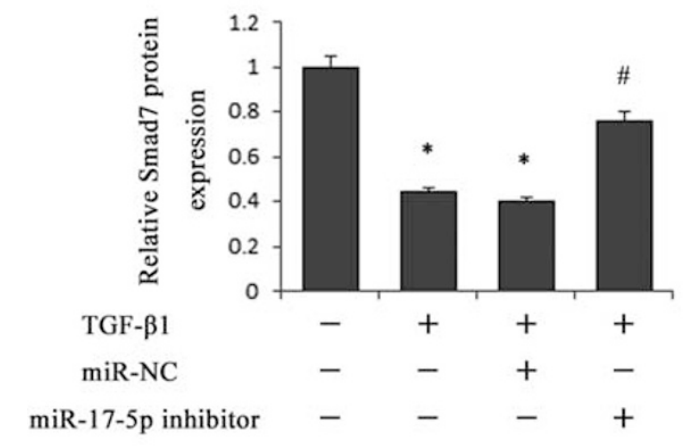

b

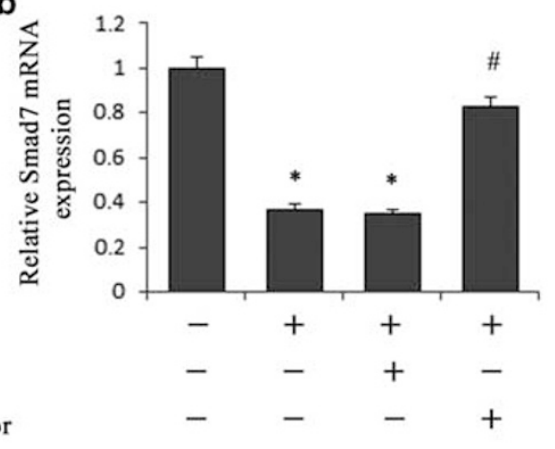

C

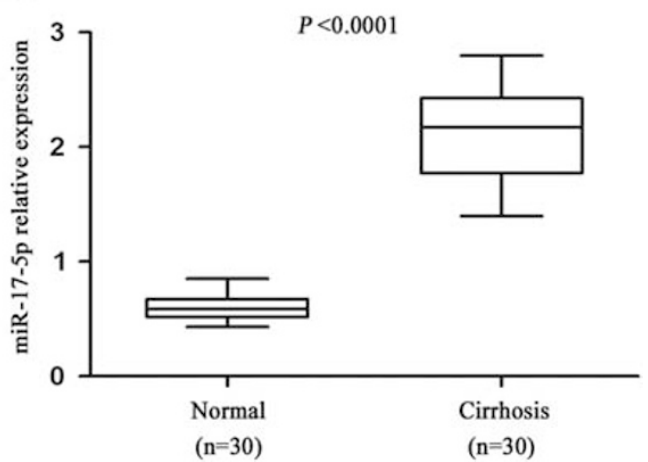

Figure 6 miR-17-5p modulates Smad7 expression and is increased in serum of patients with liver cirrhosis. (a) Suppression of Smad7 protein by TGF- $\beta 1$ was restored by miR-17-5p inhibitor. $\beta$-actin was used as the internal control for western blot analysis. (b) Suppression of Smad7 mRNA expression by TGF- $\beta 1$ was restored by miR-17-5p inhibitor. Each value represents the mean \pm s.d. of three experiments. ${ }^{*} P<0.05$, compared with the control. ${ }^{\#} P<0.05$, compared with the TGF- $\beta 1$ group. (c) Serum levels of miR-17-5p were significantly higher in patients with cirrhosis, compared to healthy controls $(P<0.0001)$.

the principal cell type responsible for synthesis and deposition of ECM proteins. ${ }^{2}$ Transfection of miR-17-5p inhibitor led to significant downregulation of type I collagen and $\alpha$-SMA, a marker of HSC activation, induced by TGF- $\beta 1$. To our knowledge, this is the first report to show that miR-17-5p is involved in TGF- $\beta 1$-induced HSC proliferation and activation.

TGF- $\beta 1$ is an important profibrogenic cytokine that mainly activates HSCs through the TGF- $\beta 1 /$ Smad signal pathway. ${ }^{27}$ Activation of TGF- $\beta 1 /$ Smad signaling is a key mechanism of liver fibrosis. After binding to its receptors, TGF- $\beta 1$ activates downstream signal transduction pathways, including Smad2 and $\mathrm{Smad} 3$, to mediate fibrosis, which is negatively regulated by Smad7, an inhibitor of TGF- $\beta 1$ signaling. ${ }^{28}$ Phosphorylation of Smad2 and Smad3 can be blocked by Smad7. For instance, a study by Dooley et al. showed that overexpression of Smad7 attenuates TGF- $\beta$-mediated fibrogenesis and protects against liver damage through inhibiting Smad2/3 phosphorylation. ${ }^{29}$ Therefore, Smad7 acts as a negative regulator of HSC activation and hepatic fibrosis, both in vitro and in vivo. ${ }^{29}$ Loss of Smad7 has been reported in fibrotic liver and during HSC activation induced by TGF- $\beta 1 .{ }^{26}$ Previous studies have additionally shown that loss of Smad7 expression results from epigenetic modifications, including microRNA, histone modification and promoter hypermethylation. ${ }^{30-32}$ Data from the current investigation disclosed significant suppression of both Smad7 mRNA and protein expression by TGF- $\beta 1$, which could be blocked by the miR-17-5p inhibitor. The targets of miRNAs may differ due to variations between rat and human tissues. For example, miR-29b was shown to induce global DNA hypomethylation in human acute myeloid leukemia cells through direct targeting of DNMT3a and DNMT3b and indirect targeting of DNMT1. ${ }^{33}$ However, our group identified only DNMT3b as a target of miR-29b in rat HSCs. ${ }^{34}$ Therefore, the targets of miRNAs may vary in different tissues, and should be determined using confirmatory analyses, such as the dual luciferase reporter experiment. Here, Smad7 was predicted as a target of miR-17-5p, which was confirmed with the dual luciferase reporter experiment. Our results suggest that miR-17-5p promotes HSC-associated proliferation, ECM production, transdifferentiation, at least in part, via reduction of Smad7 expression. However, the functions of miR-17-5p in HSC may be more complex than previously assumed, and further in vivo functional studies are required to establish its specific role in liver fibrosis.

Accumulating studies have demonstrated that miRNAs are utilizable not only as a potential tool for diagnosis of liver fibrosis but also a novel fibrosis biomarker. ${ }^{35}$ Since it is difficult to obtain liver tissue, miRNAs in the tissue do not generally serve as biomarkers. miRNAs are additionally found 
in body fluids, including serum/plasma. ${ }^{36}$ However, the specific roles of serum/plasma miRNAs in regulating HSC activation are currently unclear. Serum miR-122 has been identified as an independent marker for predicting survival of patients with liver cirrhosis. ${ }^{37}$ Huang et al. reported that serum miR-33a levels are positively associated with the progression of hepatic fibrosis. ${ }^{38}$ In the current study, we showed that serum miR-17-5p levels are increased in patients with cirrhosis, compared to healthy controls, indicating that miR-17-5p may be effectively developed as a clinical diagnostic marker. However, the sample size was small, and experiments with large sample cohorts are needed to further validate the utility of this marker.

In conclusion, our results have provided new insights showing that miR-17-5p contributes to HSC proliferation and activation, at least in part, via targeting Smad7. Moreover, the increased levels of serum miR-17-5p in patients with cirrhosis support its potential utility as a diagnostic biomarker for the disease.

\section{ACKNOWLEDGMENTS}

The project was supported by National Natural Science Foundation of China (81000176/H0317, 81100292/H0317), Zhejiang Provincial Natural Science Foundation of China (Y2090326, Y2110634), Wang Bao-En Liver Fibrosis Foundation (No.20100002, 20120127) and Wenzhou Municipal Science and Technology Bureau (Y20120127).

\section{DISCLOSURE/CONFLICT OF INTEREST}

The authors declare no conflict of interest.

1. Sun $X, \mathrm{He} Y, M a T$ et al. Participation of miR-200a in TGF-beta1mediated hepatic stellate cell activation. Mol Cell Biochem 2014;388: $11-23$.

2. He Y, Huang C, Sun X et al. MicroRNA-146a modulates TGF-beta1induced hepatic stellate cell proliferation by targeting SMAD4. Cell Signal 2012;24:1923-1930.

3. Wei J, Feng L, Li Z et al. MicroRNA-21 activates hepatic stellate cells via PTEN/Akt signaling. Biomed Pharmacother 2013:67:387-392.

4. Friedman SL. Evolving challenges in hepatic fibrosis. Nat Rev Gastroenterol Hepatol 2010;7:425-436.

5. Friedman SL. Hepatic stellate cells: protean, multifunctional, and enigmatic cells of the liver. Physiol Rev 2008;88:125-172.

6. Dooley S, Delvoux B, Streckert M et al. Transforming growth factor beta signal transduction in hepatic stellate cells via Smad2/3 phosphorylation, a pathway that is abrogated during in vitro progression to myofibroblasts. TGFbeta signal transduction during transdifferentiation of hepatic stellate cells. FEBS Lett 2001;502:4-10.

7. Bataller R, Brenner DA. Liver fibrosis. J Clin Invest 2005;115:209-218.

8. Carthew RW, Sontheimer EJ. Origins and Mechanisms of miRNAs and siRNAs. Cell 2009;136:642-655.

9. Schickel R, Boyerinas B, Park SM et al. MicroRNAs: key players in the immune system, differentiation, tumorigenesis and cell death. Oncogene 2008;27:5959-5974.

10. Zhao J, Tang N, Wu K et al. MiR-21 Simultaneously Regulates ERK1 Signaling in HSC Activation and Hepatocyte EMT in Hepatic Fibrosis. PLoS One 2014;9:e108005.

11. Sekiya $Y$, Ogawa $T$, Yoshizato $K$ et al. Suppression of hepatic stellate cell activation by microRNA-29b. Biochem Biophys Res Commun 2011;412:74-79.

12. Zheng J, Wu C, Lin Z et al. Curcumin up-regulates phosphatase and tensin homologue deleted on chromosome 10 through microRNAmediated control of DNA methylation - a novel mechanism suppressing liver fibrosis. FEBS J. 2014;281:88-103.

13. Olive V, Jiang I, He L. mir-17-92, a cluster of miRNAs in the midst of the cancer network. Int J Biochem Cell Biol 2010;42:1348-1354.
14. Yang $F$, Yin $Y$, Wang $F$ et al. miR-17-5p Promotes migration of human hepatocellular carcinoma cells through the p38 mitogen-activated protein kinase-heat shock protein 27 pathway. Hepatology 2010;51: 1614-1623.

15. Shan SW, Fang L, Shatseva $T$ et al. Mature miR-17-5p and passenger miR-17-3p induce hepatocellular carcinoma by targeting PTEN, GalNT7 and vimentin in different signal pathways. J Cell Sci 2013;126: $1517-1530$.

16. Zheng J, Dong P, Gao $S$ et al. High expression of serum miR-17-5p associated with poor prognosis in patients with hepatocellular carcinoma. Hepatogastroenterology 2013;60:549-552.

17. Yao QY, Xu BL, Wang JY et al. Inhibition by curcumin of multiple sites of the transforming growth factor-beta1 signalling pathway ameliorates the progression of liver fibrosis induced by carbon tetrachloride in rats. BMC Complement Altern Med 2012;12:156.

18. Zheng J, Wu C, Lin Z et al. Curcumin up-regulates phosphatase and tensin homologue deleted on chromosome 10 through microRNAmediated control of DNA methylation - a novel mechanism suppressing liver fibrosis. FEBS J 2014;281:88-103.

19. Dai W, Zhao J, Tang $\mathrm{N}$ et al. MicroRNA-155 attenuates activation of hepatic stellate cell by simultaneously preventing EMT process and ERK1 signalling pathway. Liver Int 2014;35:1234-1243.

20. Schmittgen TD, Livak KJ. Analyzing real-time $P C R$ data by the comparative C(T) method. Nat Protoc 2008;3:1101-1108.

21. Tu X, Zhang H, Zhang J et al. MicroRNA-101 suppresses liver fibrosis by targeting the TGFbeta signalling pathway. J Pathol 2014;234:46-59.

22. Lin J, Chen A. Activation of peroxisome proliferator-activated receptorgamma by curcumin blocks the signaling pathways for PDGF and EGF in hepatic stellate cells. Lab Invest 2008;88:529-540.

23. Chen $X, B a Y, M a L$ et al. Characterization of microRNAs in serum: a novel class of biomarkers for diagnosis of cancer and other diseases. Cell Res 2008;18:997-1006.

24. Fang $C$, Zhu DX, Dong $\mathrm{HJ}$ et al. Serum microRNAs are promising novel biomarkers for diffuse large B cell lymphoma. Ann Hematol 2012;91: 553-559.

25. He Y, Huang C, Zhang SP et al. The potential of microRNAs in liver fibrosis. Cell Signal 2012;24:2268-2272.

26. Bian EB, Huang $\mathrm{C}$, Wang $\mathrm{H}$ et al. Repression of Smad7 mediated by DNMT1 determines hepatic stellate cell activation and liver fibrosis in rats. Toxicol Lett 2014;224:175-185.

27. Friedman SL. Liver fibrosis: from mechanisms to treatment. Gastroenterol Clin Biol 2007;31:812-814.

28. Derynck R, Zhang YE. Smad-dependent and Smad-independent pathways in TGF-beta family signalling. Nature 2003;425:577-584.

29. Dooley S, Hamzavi J, Ciuclan L et al. Hepatocyte-specific Smad7 expression attenuates TGF-beta-mediated fibrogenesis and protects against liver damage. Gastroenterology 2008;135:642-659.

30. Xia H, Ooi LL, Hui KM. MicroRNA-216a/217-induced epithelialmesenchymal transition targets PTEN and SMAD7 to promote drug resistance and recurrence of liver cancer. Hepatology 2013;58:629-641.

31. Glenisson W, Castronovo V, Waltregny D. Histone deacetylase 4 is required for TGFbeta1-induced myofibroblastic differentiation. Biochim Biophys Acta 2007;1773:1572-1582.

32. Matsumura N, Huang Z, Mori $S$ et al. Epigenetic suppression of the TGF-beta pathway revealed by transcriptome profiling in ovarian cancer. Genome Res 2011;21:74-82.

33. Garzon R, Liu S, Fabbri $M$ et al. MicroRNA-29b induces global DNA hypomethylation and tumor suppressor gene reexpression in acute myeloid leukemia by targeting directly DNMT3A and $3 B$ and indirectly DNMT1. Blood 2009;113:6411-6418.

34. Zheng J, Wu C, Lin Z et al. Curcumin up-regulates phosphatase and tensin homologue deleted on chromosome 10 through microRNAmediated control of DNA methylation-a novel mechanism suppressing liver fibrosis. FEBS J 2014;281:88-103.

35. Ogawa T, Enomoto M, Fujii $\mathrm{H}$ et al. MicroRNA-221/222 upregulation indicates the activation of stellate cells and the progression of liver fibrosis. Gut 2012;61:1600-1609.

36. Weber JA, Baxter DH, Zhang $\mathrm{S}$ et al. The microRNA spectrum in 12 body fluids. Clin Chem 2010;56:1733-1741.

37. Waidmann O, Koberle V, Brunner $\mathrm{F}$ et al. Serum microRNA-122 predicts survival in patients with liver cirrhosis. PLoS One 2012;7:e45652.

38. Huang CF, Sun CC, Zhao F et al. miR-33a levels in hepatic and serum after chronic HBV-induced fibrosis. J Gastroenterol 2014;50:480-490. 